\title{
The UK case-control study of cerebral oedema complicating diabetic ketoacidosis in children
}

\author{
J. A. Edge $・$ R. W. Jakes $\cdot$ Y. Roy $\cdot$ M. Hawkins • \\ D. Winter $\cdot$ M. E. Ford-Adams • N. P. Murphy • \\ A. Bergomi • B. Widmer • D. B. Dunger
}

Received: 31 January 2006 / Accepted: 25 May 2006 / Published online: 18 July 2006

(C) Springer-Verlag 2006

\begin{abstract}
Aims/hypothesis Cerebral oedema complicating diabetic ketoacidosis (DKA) remains the major cause of morbidity and mortality in children with type 1 diabetes, but its aetiology remains unknown. Our objective was to determine the impact of baseline biochemical factors and of treatment-related variables on risk of the development of cerebral oedema in children with DKA.

Materials and methods This was a national UK casecontrol study. Through the British Paediatric Surveillance Unit we identified 43 cases of cerebral oedema. Through a parallel reporting system, we also identified 2,940 episodes of DKA and selected 169 control subjects on the basis of comparable age, sex, numbers of new or known cases of diabetes and date of admission. Baseline biochemical data and treatment-related variables were extracted from the clinical notes of cases and control subjects.

Results Allowing for differences in age, sex and new or known diabetes, cases were more acidotic at diagnosis of
\end{abstract}

J. A. Edge $\cdot$ Y. Roy $\cdot$ M. E. Ford-Adams $\cdot$ N. P. Murphy $\cdot$

A. Bergomi

Department of Paediatrics, John Radcliffe Hospital,

Oxford, UK

B. Widmer $\cdot$ D. B. Dunger $(\triangle)$

Department of Paediatrics, Addenbrooke's NHS Trust,

Hills Road, Box 116, Cambridge CB2 2QQ, UK

e-mail: dbd25@cam.ac.uk

M. Hawkins $\cdot$ D. Winter

Department of Public Health and Epidemiology,

University of Birmingham,

Birmingham, UK

R. W. Jakes

MRC Epidemiology Unit, Elsie Widdowson Laboratory,

Cambridge, UK
DKA (odds ratio [OR] for events in the least acidotic compared with the most acidotic tertile $=0.02[95 \%$ CI: $0.002-0.15], p<0.001)$. In addition, cases had higher potassium and urea levels at baseline. Calculated osmolality and baseline glucose were not significantly different. After allowing for severity of acidosis, insulin administration in the first hour (OR 12.7 [1.41-114.5], $p=0.02$ ) and volume of fluid administered over the first $4 \mathrm{~h}$ (OR 6.55 [1.38-30.97], $p=0.01$ ) were associated with risk. Low baseline plasma sodium and an elevated $\mathrm{p}_{\mathrm{a}} \mathrm{CO}_{2}$ also contributed to risk in the final regression model. Bicarbonate administration was not associated with increased risk of an event when corrected for acidosis.

Conclusions/interpretation In this case-control study of DKA, baseline acidosis and abnormalities of sodium, potassium and urea concentrations were important predictors of risk of cerebral oedema. Additional risk factors identified were early administration of insulin and high volumes of fluid. These observations should be taken into account when designing treatment protocols.

Keywords Cerebral oedema . Children .

Diabetic ketoacidosis

$\begin{array}{ll}\text { Abbreviations } \\ \text { BPSU } & \text { British Paediatric Surveillance Unit } \\ \text { DKA } & \text { diabetic ketoacidosis } \\ \text { OR } & \text { odds ratio }\end{array}$

\section{Introduction}

Cerebral oedema is a devastating and generally unpredictable complication of diabetic ketoacidosis (DKA) in children 
with type 1 diabetes. In the UK around 70 to $80 \%$ of diabetes-related deaths in children under 12 years of age are the result of cerebral oedema [1]. The mortality associated with cerebral oedema is around 25 to $30 \%$ and 35 to $40 \%$ of survivors are left with major neurological deficits $[2,3]$. However, the aetiology of cerebral oedema complicating DKA is still unknown. Examination of series of cases of cerebral oedema has led to speculation that possible contributing factors may be a high initial plasma glucose concentration [4], excessive rates of i.v. fluid administration [5] and failure of plasma sodium levels to rise as glucose levels fall during treatment $[6,7]$. Yet the only sizeable casecontrol study reported that the only factors that predicted the development of cerebral oedema were a lower $\mathrm{pCO}_{2}$ and a higher plasma urea concentration at presentation [3]. The only associated treatment modality was the use of bicarbonate; fluid rates and sodium content of fluid administered were no different between cases and controls [3]. However, that study was based on data from selected hospital populations and did not prospectively identify cases and controls from a population base.

In order to identify further factors in the clinical presentation and management of children with DKA possibly associated with the development of cerebral oedema, we carried out a large national case-control study. The initial incidence and outcome data have already been reported [2] and we now report analysis of baseline biochemical data and treatment-related variables from confirmed cases and controls.

\section{Subjects, materials and methods}

\section{Subjects}

\section{Ascertainment of cases of cerebral oedema}

All paediatricians involved in the care of children with type 1 diabetes in England, Scotland and Wales were asked to report any cases of possible cerebral oedema or any death during the assessment or treatment of DKA in children to the British Paediatric Surveillance Unit (BPSU) over a 3 -year period [2]. The BPSU coordinates a reporting scheme whereby all consultant paediatricians in the UK are sent a monthly reporting card containing a list of specific conditions, providing active surveillance of certain rare childhood disorders. On average, $94 \%$ of BPSU clinicians return the report card each month (http://bpsu.inopsu.com).

The criteria for reporting a case for this study were: (1) age $<16$ years; (2) sudden or unexpected deterioration in conscious level in a child with DKA; and (3) any death during assessment or management of DKA. Sixty potential cases were reported over a 3-year period [2].

\section{Identification of controls}

In order to ascertain episodes of DKA that could provide control data, a separate national reporting system was established [2]. Two hundred and forty-three consultants in 231 hospitals in England, Scotland and Wales reported episodes of DKA monthly for the 2 years in the middle of the cerebral oedema case-collection period. The definition of DKA was: 'decompensated diabetes mellitus with evidence of ketoacidosis $(\mathrm{pH}<7.3$ or plasma bicarbonate level $<18 \mathrm{mmol} / \mathrm{l}$ or heavy ketonuria)'. Data requested about each episode of DKA were: date of birth, sex, date of episode and whether the child had newly diagnosed ('new') or 'known' diabetes. Two thousand nine hundred and forty admissions with DKA were reported.

\section{Ethics}

The study was approved by the Central Oxford Research Ethics Committee and by other local ethics committees where submission was considered necessary. There was no contact with patients or their families.

Methods

\section{Validation of cases}

Sixty potential cases were reported and the medical notes were examined. A case of cerebral oedema was confirmed when a deterioration in the level of consciousness was accompanied by one or more further signs of raised intracranial pressure (hypertension and bradycardia, breathing pattern abnormalities, pupillary abnormalities, squint, blurred disc margins, decerebrate or decorticate posturing, respiratory arrest or radiological or post-mortem evidence of moderate to severe cerebral oedema). Forty-three cases of cerebral oedema were confirmed after examination of the case records. The 17 episodes without definite cerebral oedema had either no evidence from the records of reduction in conscious level at all or a mild reduction with no other evidence of raised intracranial pressure, and all recovered rapidly and fully.

\section{Selection of controls}

One hundred and sixty-nine controls were selected from the 2,940 reported episodes of DKA on the basis of age (age groups $0-1,2-4,5-10,11-15$ years), sex and whether the patients had new or known diabetes. In order to remove any potential bias resulting from a possible systematic change in the management of DKA over the 2-year period, we also matched cases and controls by month of admission to within a 6-month period of the presentation of the case of 
cerebral oedema. A research assistant visited hospitals to examine the medical records of controls. In total, it was possible to match 169 controls with the 43 cases, approximately four controls per case.

\section{Data collection and database}

Details of demographic characteristics, initial biochemical data, treatment regimens and changes in biochemical data during treatment were retrieved from the hospital notes of cases and controls and entered into an Access database.

Initial biochemical data were defined as the first records before treatment was initiated. Data relating to degree of acidosis were variably reported as arterial, capillary or venous $\mathrm{pH}$, or bicarbonate. There were no differences in the relationships between the three variables whether arterial, capillary or venous data were used, so all were included. Where both $\mathrm{pH}$ and bicarbonate were available they were highly correlated. We therefore derived an index of 'acidosis' from the distribution of $\mathrm{pH}$ and $\mathrm{HCO}_{3}$ in cases and controls. Where $\mathrm{pH}$ was available, 'acidosis' was defined by $\mathrm{pH}$ tertile, otherwise tertile of $\mathrm{HCO}_{3}$ was used to define severity of 'acidosis'. With respect to venous $\mathrm{pCO}_{2}$ values, these were arterialised using the formula: venous $\mathrm{pCO}_{2}-6=$ arterial $\mathrm{pCO}_{2}\left(\mathrm{p}_{\mathrm{a}} \mathrm{CO}_{2} \mathrm{~mm} / \mathrm{Hg}\right)$. Corrected sodium was calculated using the formula: corrected sodium=plasma sodium $+(1.43 \times$ blood glucose -5$) / 5$. Estimated plasma osmolality was calculated using the formula: osmolality $=(2 \times$ plasma sodium)+plasma urea+plasma glucose (all mmol/l).

Cerebral oedema in the cases was evident at admission in five of the 43 cases. Median time to event of the remaining 38 patients was $7 \mathrm{~h}$ after admission (range: $1-24 \mathrm{~h}$ ).

\section{Data analysis}

Comparisons of means of biochemical data at baseline were first undertaken using multiple linear regression models, adjusting for the principal matching variables (age, sex, and whether the patient had 'new' or 'known' diabetes). In order to determine whether differences in baseline variables were linear, we used logistic regression, examining quartiles or tertiles with tests of heterogeneity, linear trend and non-linearity.

In order to identify potential treatment-related determinants of risk of cerebral oedema, we carried out multivariate modelling, allowing for matching variables (age, sex and whether the patient had new or known diabetes). We also included baseline acidosis in these analyses because of its major influence on risk of an event. Linearity of effect was not assumed and treatment variables were divided into quartiles or tertiles and tests for heterogeneity, linear trend and non-linearity were used. For the estimation of relative risks associated with volume of fluid the cumulative volume of fluid administered over the first $1-4 \mathrm{~h}$ was categorised into tertiles. Insulin, administered i.v. during the first hour, was categorised into those who did not receive insulin and those who did. In the analysis of insulin dose over the first $2 \mathrm{~h}$ patients were categorised into tertiles according to the dose of insulin. Administration of bicarbonate was analysed as a dichotomised variable.

Rate of change in each measured biochemical variable from initial assessment after admission to the diagnosis of cerebral oedema in a case and for an identical interval from initial measurement of cerebral oedema in the matched controls was determined using simple linear regression models for repeated measures [8]. All available data from each subject were fitted for available time-points and compared between cases and controls.

Finally, important baseline biochemical and treatmentrelated variables were combined in an unconditional stepwise logistic regression models. Logistic regression models were confined to those subjects where all data were available.

Level of significance was at the $p<0.05$ level and all analyses were undertaken using Stata (Stata Statistical Software Release 8.0; Stata Corporation, College Station, TX, USA).

\section{Results}

Baseline characteristics

The baseline characteristics and first recorded biochemical variables before treatment of cases and controls are shown in Table 1. Differences in baseline variables were assessed following adjustment for age, sex and new or known diabetes. Cases were significantly more acidotic than controls. $\mathrm{p}_{\mathrm{a}} \mathrm{CO}_{2}$ levels were lower in cases, but this did not reach statistical significance. Plasma concentrations of potassium and urea were also significantly higher in cases than in controls. Calculated osmolality, sodium levels, corrected sodium and baseline glucose levels were not significantly different between cases and controls.

The results of logistic regression analyses by quartile or tertile distribution of these biochemical analyses are summarised in Table 2. Degree of acidosis, as assessed by $\mathrm{pH}$, bicarbonate or a combined measure (combined from tertile of bicarbonate and $\mathrm{pH}$ ) remained highly significant. Potassium and urea both showed associations, with the risk of cerebral oedema being particularly apparent in the highest quartiles of distribution. There were trends for increasing risk with lower baseline sodium concentrations and with lower $\mathrm{p}_{\mathrm{a}} \mathrm{CO}_{2}$, but these were not statistically significant. 
Table 1 Characteristics of the cases and controls at presentation with DKA

\begin{tabular}{|c|c|c|c|c|c|}
\hline & \multicolumn{2}{|c|}{ Cases } & \multicolumn{2}{|c|}{ Controls } & \multirow[t]{2}{*}{$p$ value $^{\mathrm{a}}$} \\
\hline & $n$ & Value & $n$ & Value & \\
\hline Age (years) & 43 & $8.5(4.5)$ & 169 & $8.9(4.3)$ & $<0.6$ \\
\hline Male sex $(\%)$ & 43 & $17(39.5 \%)$ & 169 & $54(31.9 \%)$ & $<0.4$ \\
\hline Newly diagnosed diabetes & 43 & $24(55.8 \%)$ & 169 & $94(55.6 \%)$ & $<1.0$ \\
\hline \multicolumn{6}{|l|}{ Biochemistry } \\
\hline Sodium (mmol/l) & 37 & $132.6(5.2)$ & 150 & $134.6(5.6)$ & $<0.1$ \\
\hline Potassium (mmol/l) & 33 & $4.9(1.0)$ & 145 & $4.5(0.7)$ & $<0.01$ \\
\hline Urea $(\mathrm{mmol} / \mathrm{l})$ & 36 & $7.8(3.0)$ & 142 & $6.5(2.7)$ & $<0.02$ \\
\hline Bicarbonate $(\mathrm{mmol} / \mathrm{l})$ & 39 & $5.7(3.7)$ & 140 & $9.6(4.4)$ & $<0.001$ \\
\hline Calculated osmolality (mOsmol/kg) & 32 & $305(15)$ & 139 & $304(14)$ & $<0.8$ \\
\hline Corrected sodium $(\mathrm{mmol} / \mathrm{l})$ & 33 & $140(6)$ & 144 & $141(6)$ & $<0.3$ \\
\hline Glucose $(\mathrm{mmol} / \mathrm{l})$ & 37 & $30.8(8.3)$ & 146 & $29.0(10.3)$ & $<0.3$ \\
\hline $\mathrm{pH}$ & 36 & $7.0(0.1)$ & 111 & $7.2(0.1)$ & $<0.001$ \\
\hline $\mathrm{p}_{\mathrm{a}} \mathrm{CO}_{2}(\mathrm{mmHg})^{\mathrm{b}}$ & 31 & $16.9(8.2)$ & 96 & $20.7(8.0)$ & $<0.07$ \\
\hline
\end{tabular}

All values means (SD), except male sex and newly diagnosed diabetes (\%)

${ }^{a}$ Differences ( $p$ value) in clinical and continuously distributed biochemical parameters between cases and controls are adjusted for age, sex and new or known diabetes case where appropriate

${ }^{b} \mathrm{kPa}$ were converted to $\mathrm{mmHg}$ prior to venous $\mathrm{pCO}_{2}$ values being arterialised using the formula: venous $\mathrm{pCO}_{2}-6=\mathrm{arterial}_{\mathrm{a}} \mathrm{CO}_{2}(\mathrm{mmHg})$

Treatment variables

\section{Fluid volume}

The cumulative volume of i.v. fluid administered over the first 3 to $4 \mathrm{~h}$ had an additional significant effect on risk of an event after allowing for matching variables and degree of acidosis at baseline (Table 3).

\section{Sodium concentration of fluids administered and potassium replacement}

All but one of the subjects studied had i.v. solutions administered with a sodium concentration of $\geq 150 \mathrm{mmol} / \mathrm{l}$ in the first hour of treatment. During the second hour of treatment seven out of 87 controls (8\%) and two out of 31 cases $(6.5 \%)$ had solutions containing $<150 \mathrm{mmol} / \mathrm{l}$ of sodium. After adjusting for matching variables and baseline acidosis, the odds ratio (OR) for an event was $8.4(95 \%$ CI: 0.6-126), but this was not significant. No significant associations were observed between amount of potassium administered and risk of the development of cerebral oedema.

\section{Bicarbonate administration}

Bicarbonate was administered to six out of the 169 controls (3.6\%) and five of the 43 cases (12\%). The unadjusted OR for risk of cerebral oedema was 3.7 (95\% CI: 1.02-13.10, $p<0.05)$. However, this association was attenuated when adjustment was made for matching variables and baseline acidosis (OR 1.5 [95\% CI: 0.39-5.76]).

\section{Insulin replacement}

In $50 \%$ of the study group no insulin was administered in the first hour of fluid treatment. If insulin was administered in the first hour the OR for an event was 4.7 (95\% CI: $1.5-13.9, p<0.007)$ after adjustment for age, sex, new/known diabetes and baseline acidosis.

The dose of insulin administered over the first $2 \mathrm{~h}$ was also significantly associated with the risk of cerebral oedema (Table 4). These findings persisted when insulin dose was analysed as dose $/ \mathrm{kg}$ body weight $(p<0.02$ trend).

Biochemical changes over time from presentation in cases and controls

Changes in the plasma concentrations of glucose, potassium, urea, sodium and bicarbonate were analysed up until the occurrence of cerebral oedema in the cases and the comparable interval in the controls. Changes in glucose, sodium and urea over time were no different in cases and controls, but the fall in potassium levels was significantly greater in cases (mean fall: $0.15 \mathrm{mmol} \mathrm{l}^{-1} \mathrm{~h}^{-1}$ ) than in controls (mean fall $0.06 \mathrm{mmol} \mathrm{l}^{-1} \mathrm{~h}^{-1}$ ), with a $\beta$-coefficient for difference in slopes of -3.53 (95\% CI: -6.67 to -0.39 , $p<0.03$ ). However, this difference was not significant after adjustment for baseline potassium levels $(p<0.09)$.

Models combining baseline biochemical and treatment-related variables

In a logistic model combining all significant biochemical variables at admission and significant treatment variables, 
Table 2 Univariate logistic regression models between baseline biochemical variables and risk of cerebral oedema

\begin{tabular}{|c|c|c|c|c|}
\hline Tertile/quartile range & $n$ & OR & $95 \% \mathrm{CI}$ & $\begin{array}{l}p \text { for } \\
\text { trend }^{\text {a }}\end{array}$ \\
\hline \multicolumn{5}{|l|}{ Sodium (mmol/l) } \\
\hline $105-130$ & 46 & 1.00 & - & \\
\hline $131-134$ & 43 & 1.26 & $0.48-3.10$ & \\
\hline $135-137.1$ & 50 & 0.72 & $0.25-2.01$ & \\
\hline $138-149$ & 48 & 0.39 & $0.12-1.24$ & 0.07 \\
\hline \multicolumn{5}{|l|}{ Potassium (mmol/l) } \\
\hline $1.0-4.1$ & 53 & 1.00 & - & \\
\hline $4.2-4.6$ & 45 & 2.32 & $0.75-7.19$ & \\
\hline $4.7-5.1$ & 37 & 1.28 & $0.34-4.76$ & \\
\hline $5.2-7.8$ & 43 & 3.24 & $1.05-9.99$ & $<0.09$ \\
\hline \multicolumn{5}{|l|}{ Urea (mmol/l) } \\
\hline $1.8-4.7$ & 44 & 1.00 & - & \\
\hline $4.8-6.6$ & 48 & 0.77 & $0.23-2.55$ & \\
\hline $6.7-8.2$ & 41 & 1.14 & $0.33-3.87$ & \\
\hline $8.3-17.1$ & 45 & 3.00 & $1.03-8.73$ & $<0.02$ \\
\hline \multicolumn{5}{|l|}{$\mathrm{pH}$} \\
\hline $6.68-7.04$ & 51 & 1.00 & - & \\
\hline $7.05-7.20$ & 49 & 0.22 & $0.08-0.57$ & \\
\hline $7.21-7.36$ & 47 & No cases & - & $<0.001$ \\
\hline \multicolumn{5}{|l|}{ Bicarbonate (mmol/l) } \\
\hline $2.0-6.0$ & 35 & 1.00 & - & \\
\hline $6.3-10.6$ & 30 & 0.60 & $0.16-2.18$ & \\
\hline $11.0-22.7$ & 36 & 0.06 & $0.01-0.51$ & 0.004 \\
\hline \multicolumn{5}{|l|}{$\mathrm{p}_{\mathrm{a}} \mathrm{CO}_{2}(\mathrm{mmHg})$} \\
\hline $6.0-12.5$ & 29 & 1.00 & - & \\
\hline $12.8-19.5$ & 34 & 0.42 & $(0.13-1.31)$ & \\
\hline $20.3-25.4$ & 32 & 0.34 & $(0.09-1.18)$ & \\
\hline $25.5-40.5$ & 32 & 0.27 & $(0.06-1.03)$ & $<0.06$ \\
\hline \multicolumn{5}{|l|}{ Acidosis $^{\mathrm{b}}$} \\
\hline- & 74 & 1.00 & - & \\
\hline- & 56 & 0.34 & $(0.14-0.79)$ & \\
\hline- & 66 & 0.02 & $(0.002-0.15)$ & $<0.001$ \\
\hline
\end{tabular}

The OR of risk of development of cerebral oedema is compared across tertiles or quartiles for each variable, with a standardised risk of 1 for the lowest tertile/quartile

${ }^{a}$ Adjusted for age, sex and new/known diabetes

${ }^{b}$ Acidosis was defined by either tertile of venous bicarbonate or tertile of $\mathrm{pH}$ according to the availability of data

baseline acidosis remained highly significant. Low plasma sodium concentrations and an elevated $\mathrm{p}_{\mathrm{a}} \mathrm{CO}_{2}$ also contributed to the model, as did insulin treatment within the first hour (Table 5).

\section{Discussion}

The results of our case-control study of cerebral oedema complicating DKA in the UK confirm previous observations that baseline biochemical estimates of the degree of acidosis and sodium, potassium and urea concentrations are important predictors of risk. However, in addition, risk may
Table 3 Tertiles of total fluid volume administered over the first $4 \mathrm{~h}$ in cases and controls

\begin{tabular}{llll}
\hline Time-period/volume tertile & $n$ & OR $(95 \% \mathrm{CI})$ & $p$ for trend \\
\hline $0-1 \mathrm{~h}$ & & & \\
$18-171 \mathrm{ml}$ & 39 & 1.0 & \\
$175-400 \mathrm{ml}$ & 39 & $3.90(0.85-17.8)$ & \\
$440-1,800 \mathrm{ml}$ & 38 & $2.53(0.52-12.2)$ & $<0.5$ \\
$0-2 \mathrm{~h}$ & & & \\
$20-265 \mathrm{ml}$ & 45 & 1.0 & \\
$266-535 \mathrm{ml}$ & 43 & $2.28(0.58-9.02)$ & \\
$540-2,800 \mathrm{ml}$ & 46 & $3.4(0.82-14.0)$ & $<0.09$ \\
$0-3 \mathrm{~h}$ & & & \\
$40-385 \mathrm{ml}$ & 47 & 1.0 & \\
$391-708 \mathrm{ml}$ & 46 & $3.93(0.90-17.13)$ & \\
$720-3,800 \mathrm{ml}$ & 48 & $7.30(1.51-35.12)$ & $<0.02$ \\
$0-4 \mathrm{~h}$ & & & \\
$76-511$ & 44 & 1.0 & \\
$512-879$ & 44 & $3.30(0.71-15.27)$ & \\
$892-4,090$ & 45 & $6.55(1.38-30.97)$ & $<0.02$ \\
\hline
\end{tabular}

ORs for the risk of developing cerebral oedema calculated with a standardised risk of 1 for the lowest tertile

${ }^{a}$ Adjusted for age, sex and new/known diabetes and baseline acidosis

be further increased by early insulin administration and infusion of large volumes of fluids over the first $4 \mathrm{~h}$ of treatment.

A principal strength of our study was that it was prospective, with high ascertainment of cases and controls using independent national reporting systems over a defined time-period, and therefore was not selective. Many previous studies have been more selective, with the possibility of introducing bias [9-11]. Although identification of cases and controls was carried out prospectively, examination of case records was retrospective, and some of the cases and controls reported were invalid, because either the case did not have cerebral oedema or the control did not have DKA. Thus in a significant proportion of cases we had no controls available within individually matched sets. Unconditional logistic regression was therefore used in the analyses. Conditional analyses were undertaken on a reduced dataset and ORs for the principal findings in Table 5 were broadly similar but subject to wider CIs.

Table 4 Category of insulin dose administered over the first $2 \mathrm{~h}$ of treatment for DKA

\begin{tabular}{lllll}
\hline Insulin dose (U) & $n$ & OR & $95 \%$ CI & $p$ for trend \\
\hline 0 & 65 & 1 & - & \\
$0.2-1.9$ & 28 & 1.31 & $0.30-5.75$ & \\
$2.0-4.2$ & 28 & 2.66 & $0.77-9.12$ & \\
$4.4-11$ & 26 & 4.97 & $1.28-19.32$ & $<0.02$ \\
\hline
\end{tabular}

OR for risk of developing cerebral oedema calculated using a baseline of 1 for no insulin administration

${ }^{a}$ Adjusted for age, sex, new/known diabetes and baseline acidosis 
Table 5 Unconditional logistic regression model including matching variables, baseline biochemical variables and treatment-related variables in all of those cases and controls with complete data (20 cases and 47 controls)

\begin{tabular}{llll}
\hline & OR & $95 \%$ CI & $p$ value \\
\hline Age (per year) & 1.11 & $0.86-1.44$ & 0.4 \\
Female sex & 11.9 & $0.89-158$ & 0.06 \\
New diagnosis of diabetes & 48.8 & $1.62-1,472$ & 0.025 \\
Acidosis (lowest vs highest tertile) & 0.02 & $0.002-0.21$ & 0.001 \\
Blood glucose (per mmol/l) & 0.88 & $0.74-1.04$ & 0.1 \\
Sodium (uncorrected, per mmol/l) & 0.74 & $0.58-0.94$ & 0.013 \\
Potassium (per mmol/l) & 3.14 & $0.96-10.22$ & 0.057 \\
Urea (per mmol/l) & 1.68 & $0.98-2.88$ & 0.055 \\
$\mathrm{p}_{\mathrm{a}} \mathrm{CO}_{2}$ (per mmHg) & 1.20 & $1.01-1.43$ & 0.038 \\
Treated with insulin in hour 1 & 12.7 & $1.41-114.5$ & 0.023 \\
\hline
\end{tabular}

Using unconditional models to analyse matched data tends to bias away from the null value, so it is possible that some of the OR values are further from 1 than a conditional logistic model would have produced [12]. Adjustment for the matching of cases and controls by date of DKA episode was not possible, but this omission would have the effect of biasing towards the null value, i.e. conservative bias.

Previous studies have identified age at diagnosis, sex and newly diagnosed diabetes as potential risk factors for cerebral oedema complicating DKA [2, 13], but these factors were controlled for in our case-control design. Degree of acidosis and baseline plasma urea have also been identified as risk factors [3] and these have been confirmed by our study. Degree of acidosis is an important marker of severity of DKA and it was an important risk factor for cerebral oedema whether estimates relied on $\mathrm{pH}$ or bicarbonate measurements, or a combination of these measurements. $\mathrm{p}_{\mathrm{a}} \mathrm{CO}_{2}$ measurements at baseline tended to be lower in cases than in controls but this did not reach statistical significance. In the logistic regression model it was of borderline significance and in the opposite direction. Glaser et al. [3], in their case-control studies, matched subjects for $\mathrm{pH}$ at baseline and found low levels of $\mathrm{p}_{\mathrm{a}} \mathrm{CO}_{2}$ to be associated with risk of cerebral oedema. In our cases and controls, $\mathrm{pH}$ and $\mathrm{p}_{\mathrm{a}} \mathrm{CO}_{2}$ were closely correlated with no difference in the regression slope (data not shown). Single time-point measurements of $\mathrm{pH}$, bicarbonate and $\mathrm{p}_{\mathrm{a}} \mathrm{CO}_{2}$ may not reflect the dynamic changes in these variables which could determine cerebral spinal fluid $\mathrm{pH}$ and respiratory drive, but they suggest that, in contrast to baseline $\mathrm{pH}$, the relationship between $\mathrm{pH}$ and $\mathrm{p}_{\mathrm{a}} \mathrm{CO}_{2}$ levels is not an important predictor of risk of cerebral oedema.

The importance of raised plasma urea concentrations in increasing the risk of cerebral oedema was particularly strong in those subjects where the urea concentration was in the highest quartile at baseline. This may be indicative of pre-renal failure and reflect the duration and severity of insulin deficiency and fluid losses [14]. Elevation of potassium levels is also a marker of 'pre-renal failure', and was identified as a risk factor identified by our study. Low sodium concentration at baseline was also a significant predictor of cerebral oedema in the logistic regression analysis, but it is not possible to determine whether this reflects severe sodium loss or accumulation of 'free' fluid with administration of dilute oral fluids prior to admission.

Our study also provides new evidence that treatmentrelated variables might be important in determining risk of cerebral oedema. In previous studies, i.v. bicarbonate administration was reported as a risk factor for cerebral oedema $[3,15,16]$. In our study, although this observation was identified in univariate analysis, it was not significant when baseline degree of acidosis was added to the model. In the study reported by Glaser et al. [3] the importance of bicarbonate therapy only became evident after matching of cases and controls by baseline $\mathrm{pH}$. The considerable risk associated with baseline $\mathrm{pH}$ or acidosis in our case-control design did not permit matching by baseline $\mathrm{pH}$, but it seems unlikely that bicarbonate therapy per se is an independent risk for cerebral oedema in DKA.

Rate of fluid administration [9] and use of relatively dilute fluids in rehydration protocols $[6,7,11]$ have often been implicated in the aetiology of cerebral oedema complicating DKA [17]. Our data provide some support for this hypothesis in that the use of large volumes of fluid over the first 3 to $4 \mathrm{~h}$ of rehydration was significant in our models. The administration of relatively dilute fluids was also associated with high ORs for an event, but these were not significant, perhaps reflecting lack of power.

The increased risk of cerebral oedema with insulin administration in the first hour of fluid treatment and with larger insulin doses in the first $2 \mathrm{~h}$ of treatment has not been previously reported. The latter did not remain significant after adjustment for baseline variables but administration of insulin during the first hour remained significant. Failure to administer insulin during the first hour of therapy may reflect problems with venous access or an elective decision to treat only after rehydration has begun. However, the association cannot be explained by severity of acidosis as this was controlled for in the analysis.

The mechanism by which insulin administration in the first hour could increase risk of cerebral oedema is unclear, but it could relate to rapid changes in electrolytes. In particular, insulin administration is known to activate the sodium/proton exchanger mechanism, allowing influx of sodium into brain cells [18], and also to be involved in the regulation of cerebral cell volume by promoting influx of potassium, sodium and chloride into brain cells $[19,20]$. It may be that these effects are greater if they occur during the first phase of rapid rehydration when membrane electrolyte 
transport may be at its most active. Furthermore, it has recently been suggested that the subclinical cerebral oedema seen in most children with DKA [21, 22], may be consistent with a vasogenic (rather than cytotoxic) mechanism, possibly representing reperfusion of previously hypoperfused tissues with the administration of fluid during treatment [23]. The administration of larger volumes of fluid as a risk factor would also be compatible with this theory, and the effects of insulin on electrolyte transport may be additive.

Previous retrospective studies have identified rates of fall in sodium and glucose as potential risk factors for the development of cerebral oedema [4, 6, 7], but we were not able to confirm any of these previous associations. Rapid falls in sodium concentrations, as well as being an indication of excessive free water administration, may reflect salt-wasting in cases, as a consequence of the development of cerebral oedema [3]. We did, however, identify an association between rapid falls in potassium levels with the subsequent risk of cerebral oedema. These associations did not persist when adjustment for baseline potassium levels were included in models of risk, but both may be potential risk factors. The rate of fall in potassium may be a reflection of increasing dose of insulin, as both were highly correlated in our study $(r=0.58, p<0.001)$.

From our data we might conclude that severity of acidosis and high urea and potassium levels at baseline are particular risk factors for cerebral oedema and that early insulin therapy (in the first hour) and fluid replacement using large volumes and low sodium concentrations could increase that risk. Severely acidotic subjects, with evidence of pre-renal failure at baseline, may be at particular risk of developing cerebral oedema as even oral fluid rehydration may result in increased fluid accumulation in the brain, as reflected in cerebral oedema patients who present before treatment with i.v. fluids $[13,17,24]$. Thus children with these features at baseline should be considered at high risk of cerebral oedema and monitored closely. It has been suggested that, during treatment, it may be possible to identify impending cerebral oedema with careful monitoring, although this has not been proved prospectively [25].

In treated patients, early use of insulin also appears to be a risk factor as it may lead to rapid changes in electrolytes. High doses of insulin may also be a risk factor for cerebral oedema, as indicated by animal models [26, 27] and supported by our observational studies. Finally, the dangers inherent in using large volumes of relatively dilute fluids have been highlighted by many studies $[5,7,17]$. It is compatible with the 'idiogenic osmole' hypothesis of cerebral oedema risk, where relatively 'dilute' fluids may cross the blood-brain barrier more easily when the intracerebral osmolality is increased [26, 28].
Recently, the management of DKA in children has been reviewed by an international consensus group [29, 30]. Our findings are largely compatible with the decisions of that group as to how risk of cerebral oedema could be avoided. However, it is not possible from studies such as this to derive entirely 'safe' quantities of fluid and insulin, rather to suggest general principles which may be incorporated into management guidelines. Although the baseline biochemistry and management variables described may relate to risk of cerebral oedema they do not necessarily identify disease pathophysiology, and an idiosyncratic response to DKA itself, or to the treatment variables, remains a possibility.

Acknowledgements J. A. Edge, M. M. Hawkins and D. B. Dunger designed the study. J. A. Edge coordinated the study and supervised data collection and entry. Y. Roy collected the data, M. E. FordAdams, N. P. Murphy, A. Bergomi and D. Winter handled data. R. W. Jakes carried out the statistical analysis. D. B. Dunger, J. A. Edge and R. W. Jake wrote the paper. We are extremely grateful to C. Garbett, who coordinated the Oxford-based reporting system. We are indebted to all the paediatricians who reported cases and controls, and to R. Lynn and the staff at the BPSU. The study was supported by a Research Grant from Diabetes UK (formerly The British Diabetic Association). The study design, data collection, analysis, interpretation and manuscript preparation were the sole responsibility of the authors.

\section{References}

1. Edge JA, Ford-Adams ME, Dunger DB (1999) Causes of death in children with type 1 diabetes, 1990-1996. Arch Dis Child 81:318-323

2. Edge JA, Hawkins MM, Winter DL, Dunger DB (2001) The risk and outcome of cerebral oedema developing during diabetic ketoacidosis. Arch Dis Child 85:16-20

3. Glaser N, Barnett P, McCaslin I et al (2001) Risk factors for cerebral edema in children with diabetic ketoacidosis. N Engl J Med 344:264-269

4. Durr JA, Hoffman WH, Sklar AH, El Gammal T, Steinhart CM (1992) Correlates of brain edema in uncontrolled IDDM. Diabetes 41:627-632

5. Duck SC, Wyatt DT (1988) Factors associated with brain herniation in the treatment of diabetic ketoacidosis. J Pediatr 113:10-14

6. Harris GA, Fiordalisi I, Harris WL, Mosovich LL, Finberg L (1990) Minimizing the risk of brain herniation during treatment of diabetic ketoacidaemia: a retrospective and prospective study. J Pediatr 117:22-31

7. Harris GA, Fiordalisi I (1994) Physiologic management of diabetic ketoacidemia. A 5-year prospective pediatric experience in 231 episodes. Arch Pediatr Adolesc Med 148:1046-1052

8. Ghosh D, Deisher TA, Ellsworth JL (1999) Statistical methods for analyzing repeated measures. J Pharmacol Toxicol 42:157-162

9. Mahoney CP, Vlcek BW, DelAguila M (1999) Risk factors for developing brain herniation during diabetic ketoacidosis. Pediatr Neurol 21:721-727

10. Bello FA, Sotos JF (1990) Cerebral oedema in diabetic ketoacidosis in children. Lancet 336:64 
11. Hale PM, Rezvani I, Braunstein AW, Lipman TH, Martinez N, Garibaldi L (1997) Factors predicting cerebral edema in young children with diabetic ketoacidosis and new onset type I diabetes. Acta Paediatr 86:626-631

12. Breslow NE, Day NE (1980) Statistical methods in cancer research. Vol. 1: the analysis of case-control studies. IARC Scientific Publications, no. 32, WHO

13. Rosenbloom AL (1990) Intracerebral crises during treatment of diabetic ketoacidosis. Diabetes Care 13:22-33

14. Dunger DB, Edge JA (2001) Predicting cerebral oedema during diabetic ketoacidosis. N Engl J Med 344:302-303

15. Taubin H, Matz R (1968) Cerebral edema, diabetes insipidus, and sudden death during the treatment of diabetic ketoacidosis. Diabetes 17:108-109

16. Bureau MA, Begin R, Berthiaume Y, Shapcott D, Khoury K, Gagnon N (1980) Cerebral hypoxia from bicarbonate infusion in diabetic acidosis. J Pediatr 96:968-973

17. Edge JA, Dunger DB (1994) Variations in the management of diabetic ketoacidosis in children. Diabet Med 11:984-986

18. Van der Meulen JA, Klip A, Grinstein S (1987) Possible mechanisms for cerebral oedema in diabetic ketoacidosis. Lancet 2:306-308

19. Pollack AS, Arieff AI (1980) Abnormalities of cell volume regulation and their functional consequences. Am J Physiol 239: F195-F205

20. Trachtman H (1995) Cell volume regulation: a review of cerebral adaptive mechanisms and implications for clinical treatment of osmolal disturbances. I Pediatr Nephrol 5:743-750

21. Krane EJ, Rockoff MA, Wallman JK, Wolfsdorf JI (1985) Subclinical brain swelling in children during treatment of diabetic ketoacidosis. N Engl J Med 312:1147-1151
22. Hoffman WH, Steinhart CM, El Gammal T, Steele S, Cuadrado AR, Morse PK (1988) Cranial CT in children and adolescents with diabetic ketoacidosis. Am J Neuroradiol 9:733-739

23. Glaser NS, Wootton-Gorges SL, Marcin JP et al (2004) Mechanism of cerebral edema in children with diabetic ketoacidosis. J Pediatr 145:164-171

24. Lawrence SE, Cummings EA, Gaboury I, Daneman D (2005) Population-based study of incidence and risk factors for cerebral edema in pediatric diabetic ketoacidosis. J Pediatr 146:688-692

25. Muir AB, Quisling RG, Yang MC, Rosenbloom AL (2004) Cerebral edema in childhood diabetic ketoacidosis: natural history, radiographic findings, and early identification. Diabetes Care 27:1541-1546

26. Arieff AI, Kleeman CR (1973) Studies on mechanisms of cerebral edema in diabetic comas. Effects of hyperglycemia and rapid lowering of plasma glucose in normal rabbits. J Clin Invest 52:571-583

27. Tornheim PA (1981) Regional localization of cerebral edema following fluid and insulin therapy in streptozotocin-diabetic rats. Diabetes 30:762-766

28. Bohn D, Daneman D (2002) Diabetic ketoacidosis and cerebral oedema. Curr Opin Pediatr 14:287-291

29. Dunger DB, Sperling MA, Acerini CL et al (2004) European Society for Paediatric Endocrinology/Lawson Wilkins Pediatric Endocrine Society consensus statement on diabetic ketoacidosis in children and adolescents. Pediatrics 113:133-140

30. Dunger DB, Sperling MA, Acerini CL et al (2004) ESPE/IWPES consensus statement on diabetic ketoacidosis in children and adolescents. Arch Dis Child 89:188-194 